\title{
Complete Moment Convergence for Negatively Dependent Sequences of Random Variables
}

\author{
Qunying Wu and Yuanying Jiang \\ College of Science, Guilin University of Technology, Guilin 541004, China \\ Correspondence should be addressed to Yuanying Jiang; jyy@glut.edu.cn
}

Received 25 May 2016; Accepted 28 June 2016

Academic Editor: Alicia Cordero

Copyright (C) 2016 Q. Wu and Y. Jiang. This is an open access article distributed under the Creative Commons Attribution License, which permits unrestricted use, distribution, and reproduction in any medium, provided the original work is properly cited.

We study the complete moment convergence for sequences of negatively dependent identically distributed random variables with $\mathbb{E} X=0, \mathbb{E} \exp \left(|X|^{\alpha}\right)<\infty, 0<\alpha<1$, and $\mathbb{E} \exp \left(|X| \ln ^{-r}|X|\right)<\infty, r>0$. As a result, we establish the new complete moment convergence theorems.

\section{Introduction and Main Results}

Random variables $X$ and $Y$ are said to be negative quadrant dependent (NQD) if

$$
P(X \leq x, Y \leq y) \leq P(X \leq x) P(Y \leq y)
$$

for all $x, y \in \mathbb{R}$. A collection of random variables is said to be pairwise negative quadrant dependent (PNQD) if every pair of random variables in the collection satisfies (1).

It is important to note that (1) implies

$$
P(X>x, Y>y) \leq P(X>x) P(Y>y)
$$

for all $x, y \in \mathbb{R}$. Moreover, it follows that (2) implies (1) and, hence, (1) and (2) are equivalent. However, Ebrahimi and Ghosh [1] showed that (1) and (2) are not equivalent for a collection of 3 or more random variables. Accordingly, the following definition is needed to define sequences of negatively dependent random variables.

Definition 1. Random variables $X_{1}, \ldots, X_{n}$ are said to be negatively dependent (ND) if, for all real $x_{1}, \ldots, x_{n}$,

$$
\begin{aligned}
& P\left(\bigcap_{j=1}^{n}\left(X_{j} \leq x_{j}\right)\right) \leq \prod_{j=1}^{n} P\left(X_{j} \leq x_{j}\right), \\
& P\left(\bigcap_{j=1}^{n}\left(X_{j}>x_{j}\right)\right) \leq \prod_{j=1}^{n} P\left(X_{j}>x_{j}\right) .
\end{aligned}
$$

An infinite sequence of random variables $\left\{X_{n} ; n \geq 1\right\}$ is said to be ND if every finite subset $X_{1}, \ldots, X_{n}$ is ND.

Definition 2. Random variables $X_{1}, X_{2}, \ldots, X_{n}, n \geq 2$, are said to be negatively associated (NA) if, for every pair of disjoint subsets $A_{1}$ and $A_{2}$ of $\{1,2, \ldots, n\}$,

$$
\operatorname{cov}\left(f_{1}\left(X_{i} ; i \in A_{1}\right), f_{2}\left(X_{j} ; j \in A_{2}\right)\right) \leq 0,
$$

where $f_{1}$ and $f_{2}$ are increasing for every variable (or decreasing for every variable) function such that this covariance exists. A sequence of random variables $\left\{X_{i} ; i \geq 1\right\}$ is said to be NA if its every finite subfamily is NA.

The definition of PNQD is given by Lehmann [2]. The definition of NA is introduced by Joag-Dev and Proschan [3], and the concept of ND is given by Bozorgnia et al. [4]. These concepts of dependent random variables are very useful for reliability theory and applications.

It is easy to see from the definitions that NA implies ND. But Example 1.5 in $\mathrm{Wu}$ and Jiang [5] shows that ND does not imply NA. Thus, it is shown that ND is much weaker than NA. In the articles listed earlier, a number of wellknown multivariate distributions are shown to possess the ND properties. In many statistics and mechanic models, a ND assumption among the random variables in the models is more reasonable than an independent or NA assumption. Because of wide applications in multivariate statistical analysis and reliability theory, the notions of ND random variables 
have attracted more and more attention recently. A series of useful results have been established (cf. Bozorgnia et al. [4], Fakoor and Azarnoosh [6], Asadian et al. [7], Wu [5, 8], Wang et al. [9], and Liu et al. [10]). Hence, it is highly desirable and of considerable significance in the theory and application to study the limit properties of ND random variables theorems and applications.

Chow [11] first investigated the complete moment convergence, which is more exact than complete convergence. Thus, complete moment convergence is one of the most important problems in probability theory. The recent results can be found in Chen and Wang [12], Gut and Stadtmüller [13], Sung [14], Guo [15], and Qiu and Chen [16, 17]. In addition, Qiu and Chen [17] obtained complete moment convergence theorems for independent identically distributed sequences of random variables with $\mathbb{E} X=0, \mathbb{E} \exp \left(\ln ^{\alpha}|X|\right)<\infty$, $\alpha>1$. A natural question is whether there is any type of complete moment convergence theorems for $0<\alpha \leq 1$. In this paper, we study the complete moment convergence for sequences of negatively dependent identically distributed random variables with $\mathbb{E} X=0, \mathbb{E} \exp \left(|X|^{\alpha}\right)<\infty, 0<\alpha<1$, and $\mathbb{E} \exp \left(|X| \ln ^{-r}|X|\right)<\infty, r>0$. As a result, we establish the new complete moment convergence theorems.

In the following, the symbol $c$ stands for a generic positive constant which may differ from one place to another. Let $a_{n} \ll$ $b_{n}$ denote that there exists a constant $c>0$ such that $a_{n} \leq c b_{n}$ for sufficiently large $n, \ln x$ mean $\ln (\max (x, e))$, and $I$ denotes an indicator function.

Theorem 3. Let $0<\alpha<1,\left\{X, X_{n} ; n \geq 1\right\}$ be a sequence of $N D$ identically distributed random variables with partial sums $S_{n}=\sum_{i=1}^{n} X_{i}, n \geq 1$. Suppose that

$$
\begin{array}{r}
\mathbb{E} X=0, \\
\mathbb{E} \exp \left(|X|^{\alpha}\right)<\infty,
\end{array}
$$

and then

$$
\begin{array}{r}
\sum_{n=1}^{\infty} \exp \left(n^{\alpha}\right) n^{p} \mathbb{E}\left\{\max _{1 \leq k \leq n}\left|S_{k}\right|-\beta n\right\}_{+}^{q}<\infty \\
\forall \beta>1, q>0 \text { and all } p \in \mathbb{R} .
\end{array}
$$

Conversely, if (6) holds for $p=\alpha-2-q$ and some $\beta>0$, then

$$
\mathbb{E} \exp \left(\left|\frac{X}{(4 \beta)}\right|^{\alpha}\right)<\infty
$$

For $\alpha=1$, we have the following.

Theorem 4. Let $\left\{X, X_{n} ; n \geq 1\right\}$ be a sequence of $N D$ identically distributed random variables with partial sums $S_{n}=$ $\sum_{i=1}^{n} X_{i}, n \geq 1$. Suppose that

$$
\begin{aligned}
\mathbb{E} X & =0, \\
\mathbb{E} \exp \left(|X| \ln ^{-r}|X|\right) & <\infty \quad \text { for some } r>0,
\end{aligned}
$$

and then

$$
\begin{aligned}
\sum_{n=1}^{\infty} \exp \left(n \ln ^{-r} n\right) n^{p} \mathbb{E}\left\{\max _{1 \leq k \leq n}\left|S_{k}\right|-\beta n\right\}_{+}^{q}<\infty \\
\forall \beta>1, q>0 \text { and all } p \in \mathbb{R} .
\end{aligned}
$$

Conversely, if $\sum_{n=1}^{\infty} \exp \left(n \ln ^{-r} n\right) n^{-q-1} \ln ^{-r} n \mathbb{E}\left\{\max _{1 \leq k \leq n}\left|S_{k}\right|-\right.$ $\beta n\}_{+}^{q}<\infty$ for some $\beta>0$, then

$$
\mathbb{E} \exp \left(\left|\frac{X}{(4 \beta)}\right| \ln ^{-r}\left|\frac{X}{(4 \beta)}\right|\right)<\infty .
$$

Remark 5. By mimicking the analogous part in the proof of Theorem 2.1 in Qiu and Chen [17], (6) and (9) imply, respectively,

$$
\begin{array}{r}
\sum_{n=1}^{\infty} \exp \left(n^{\alpha}\right) n^{p} \mathbb{E}\left\{\sup _{1 \leq k \leq n}\left|\frac{S_{k}}{k}\right|-\beta\right\}_{+}^{q}<\infty \\
\forall \beta>1, q>0 \text { and all } p \in \mathbb{R}, \\
\sum_{n=1}^{\infty} \exp \left(n \ln ^{-r} n\right) n^{p} \mathbb{E}\left\{\sup _{1 \leq k \leq n}\left|\frac{S_{k}}{k}\right|-\beta\right\}_{+}^{q}<\infty \\
\forall \beta>1, q>0 \text { and all } p \in \mathbb{R} .
\end{array}
$$

Remark 6. Because (6) and (9) hold for all $q>0, p \in$ $\mathbb{R}$, and ND random sequences, (6) and (9) are very broad conclusions.

\section{Proofs}

The following four lemmas play important roles in the proof of our theorems.

Lemma 7 (Bozorgnia et al. [4]). Let $\left\{X_{n} ; n \geq 1\right\}$ be a sequence of ND random variables.

(i) Let $\left\{f_{n} ; n \geq 1\right\}$ be a sequence of Borel functions; all of them are monotone increasing (or all are monotone decreasing). Then, $\left\{f_{n}\left(X_{n}\right) ; n \geq 1\right\}$ is a sequence of $N D$ r.v.s.

(ii) Let $X_{1}, \ldots, X_{n}$ be nonnegative. Then,

$$
E\left(\prod_{j=1}^{n} X_{j}\right) \leq \prod_{j=1}^{n} E X_{j}
$$

In particular, let $t_{1}, \ldots, t_{n}$ be all nonnegative (or nonpositive) real numbers. Then,

$$
E\left(\exp \left(\sum_{j=1}^{n} t_{j} X_{j}\right)\right) \leq \prod_{j=1}^{n} E\left(\exp \left(t_{j} X_{j}\right)\right) .
$$

Lemma 8. Let $\left\{X, X_{i} ; i \geq 1\right\}$ be a sequence of $N D$ identically distributed random variables with $\mathbb{E} \exp (\lambda X)<\infty$ for any $\lambda>$ 0 . Assume that $\left\{a_{i} ; i \geq 1\right\}$ is a sequence of positive real numbers 
such that $a_{n} \uparrow \infty$ as $n \rightarrow \infty$. Then, for $S_{k}=\sum_{i=1}^{k} X_{i}, \lambda>0$, and a positive integer $n$,

$$
\begin{aligned}
& P\left(\max _{1 \leq k \leq n} S_{k}>a_{n}\right) \\
& \quad \leq \exp \left(-\lambda a_{n}\right) \sum_{k=1}^{n}(\mathbb{E} \exp (\lambda(X-\mathbb{E} X)))^{k} .
\end{aligned}
$$

Proof. Obviously, $\delta_{0} \hat{=} \sup \{\lambda \geq 0 ; \mathbb{E} \exp (\lambda(X-\mathbb{E} X))<\infty\}=$ $\infty$ from condition $\mathbb{E} \exp (\lambda X)<\infty$ for any $\lambda>0$. By Lemma 7(ii), (1.1) in Wang et al. [18] holds for any $\lambda \in \mathbb{R}$. Together with condition $0<a_{n} \uparrow \infty$, we know that the conditions of Theorem 2.2 in Wang et al. [18] are satisfied. Therefore, by Theorem 2.2 in Wang et al. [18], for any $\lambda>0$,

$$
\begin{aligned}
& P\left(\max _{1 \leq k \leq n} S_{k}>a_{n}\right) \\
& \quad \leq \min _{\lambda \in[0, \infty)} \exp \left(-\lambda a_{n}\right) \sum_{k=1}^{n}(\mathbb{E} \exp (\lambda(X-\mathbb{E} X)))^{k} \\
& \quad \leq \exp \left(-\lambda a_{n}\right) \sum_{k=1}^{n}(\mathbb{E} \exp (\lambda(X-\mathbb{E} X)))^{k} .
\end{aligned}
$$

Lemma 9. For any random variable $X$,

$$
\begin{aligned}
& \mathbb{E} \exp \left(|X|^{\alpha}\right)<\infty \Longleftrightarrow \sum_{n=1}^{\infty} \frac{\exp \left(n^{\alpha}\right)}{n^{1-\alpha}} P(|X|>n)<\infty \\
& \quad \text { for any } \alpha>0, \\
& \mathbb{E} \exp \left(|X| \ln ^{-r}|X|\right)<\infty \Longleftrightarrow \quad \\
& \sum_{n=2}^{\infty} \exp \left(n \ln ^{-r} n\right) \ln ^{-r} n P(|X|>n)<\infty
\end{aligned}
$$

$$
\text { for any } r>0 \text {. }
$$

Proof. Let $a_{n} \approx b_{n}$ denote that there exist constants $c_{1}>0$ and $c_{2}>0$ such that $c_{1} a_{n} \leq b_{n} \leq c_{2} a_{n}$ for sufficiently large $n$. We have

$$
\begin{aligned}
& \sum_{n=1}^{\infty} \frac{\exp \left(n^{\alpha}\right)}{n^{1-\alpha}} P(|X|>n) \\
& \quad=\sum_{n=1}^{\infty} \frac{\exp \left(n^{\alpha}\right)}{n^{1-\alpha}} \sum_{j=n}^{\infty} P(j<|X| \leq j+1) \\
& \quad=\sum_{j=1}^{\infty} P(j<|X| \leq j+1) \sum_{n=1}^{j} \frac{\exp \left(n^{\alpha}\right)}{n^{1-\alpha}} \\
& \approx \sum_{j=1}^{\infty} \exp \left(j^{\alpha}\right) \mathbb{E} I(j<|X| \leq j+1)
\end{aligned}
$$

$$
\begin{aligned}
& \approx \sum_{j=1}^{\infty} \mathbb{E} \exp \left(|X|^{\alpha}\right) I(j<|X| \leq j+1) \\
& \approx \mathbb{E} \exp \left(|X|^{\alpha}\right),
\end{aligned}
$$

and it follows that (17) holds.

Note that

$$
\begin{gathered}
\sum_{n=2}^{j} \exp \left(n \ln ^{-r} n\right) \ln ^{-r} n \approx \int_{2}^{j} \exp \left(x \ln ^{-r} x\right) \ln ^{-r} x \mathrm{~d} x \\
\sim \int_{2}^{j} \exp \left(x \ln ^{-r} x\right)\left(\ln ^{-r} x-r \ln ^{-r-1} x\right) \mathrm{d} x \\
\quad=\int_{2}^{j}\left(\exp \left(x \ln ^{-r} x\right)\right)^{\prime} \mathrm{d} x \approx \exp \left(j \ln ^{-r} j\right),
\end{gathered}
$$

and hence, using similar methods used to prove (17), we can prove that (18) holds.

Lemma 10. Let $\left\{X_{n} ; n \geq 1\right\}$ be a sequence of $N D$ random variables. Then, for any $x \geq 0$, there exists a positive constant $c$ such that, for all $n \geq 1$,

$$
\begin{aligned}
(1 & \left.-P\left(\max _{1 \leq k \leq n}\left|X_{k}\right|>x\right)\right)^{2} \sum_{k=1}^{n} P\left(\left|X_{k}\right|>x\right) \\
& \leq c P\left(\max _{1 \leq k \leq n}\left|X_{k}\right|>x\right) .
\end{aligned}
$$

Further, if $P\left(\max _{1 \leq k \leq n}\left|X_{k}\right|>x\right) \rightarrow 0$ as $n \rightarrow \infty$, then there exists a positive constant $c$ such that, for all $n \geq 1$,

$$
\sum_{k=1}^{n} P\left(\left|X_{k}\right|>x\right) \leq c P\left(\max _{1 \leq k \leq n}\left|X_{k}\right|>x\right) .
$$

Proof. Obviously, ND implies pairwise negative quadrant dependent (PNQD) from the definitions of ND and PNQD. Thus, Lemma 10 holds from Lemma 1.4 of Wu [19].

Proof of Theorem 3. Note that

$$
\begin{aligned}
& \mathbb{E}\left\{\max _{1 \leq k \leq n}\left|S_{k}\right|-\beta n\right\}_{+}^{q} \\
&=\beta^{q} \int_{0}^{n} q x^{q-1} P\left(\max _{1 \leq k \leq n}\left|S_{k}\right|-\beta n>\beta x\right) \mathrm{d} x \\
& \quad+\beta^{q} \int_{n}^{\infty} q x^{q-1} P\left(\max _{1 \leq k \leq n}\left|S_{k}\right|-\beta n>\beta x\right) \mathrm{d} x \\
& \ll n^{q} P\left(\max _{1 \leq k \leq n}\left|S_{k}\right|>\beta n\right) \\
&+\int_{n}^{\infty} x^{q-1} P\left(\max _{1 \leq k \leq n}\left|S_{k}\right|>\beta x\right) \mathrm{d} x .
\end{aligned}
$$


Hence, in order to establish (6), it suffices to prove, for any $p \in \mathbb{R}$,

$$
\begin{array}{r}
\sum_{n=1}^{\infty} \exp \left(n^{\alpha}\right) n^{p} P\left(\max _{1 \leq k \leq n}\left|S_{k}\right|>\beta n\right)<\infty \\
\sum_{n=1}^{\infty} \exp \left(n^{\alpha}\right) n^{p} \int_{n}^{\infty} x^{q-1} P\left(\max _{1 \leq k \leq n}\left|S_{k}\right|>\beta x\right) \mathrm{d} x<\infty
\end{array}
$$

Firstly, we prove (24). Let $\beta>1$ be arbitrary; define, for $1 \leq$ $k \leq n$,

$$
\begin{aligned}
X^{\prime}= & -\beta n I(X<-\beta n)+X I(|X| \leq \beta n) \\
& +\beta n I(X>\beta n), \\
X_{k}^{\prime}= & -\beta n I\left(X_{k}<-\beta n\right)+X_{k} I\left(\left|X_{k}\right| \leq \beta n\right) \\
& +\beta n I\left(X_{k}>\beta n\right), \\
S_{n}^{\prime}= & \sum_{k=1}^{n} X_{k}^{\prime} .
\end{aligned}
$$

It is easy to get

$$
\begin{aligned}
P\left(\max _{1 \leq k \leq n}\left|S_{k}\right|>n \beta\right) \leq & P\left(\max _{1 \leq k \leq n}\left|S_{k}^{\prime}\right|>n \beta\right) \\
& +n P(|X|>n \beta) .
\end{aligned}
$$

From (5) and the Markov inequality,

$$
\begin{aligned}
P(|X|>n \beta) & \leq \exp \left(-\beta^{\alpha} n^{\alpha}\right) \mathbb{E} \exp \left(|X|^{\alpha}\right) \\
& \ll \exp \left(-\beta^{\alpha} n^{\alpha}\right) .
\end{aligned}
$$

Set $\widetilde{X}=X^{\prime}-\mathbb{E} X^{\prime}$; then $\mathbb{E} \widetilde{X}=0$; using the obvious inequality $e^{y} \leq 1+y+\left(1+e^{y} I(y>0)\right) y^{2} / 2$, it follows that, for any $\lambda>0$,

$$
\begin{aligned}
\mathbb{E} \exp (\lambda \widetilde{X}) & \leq 1+\frac{\lambda^{2}}{2} \mathbb{E} \widetilde{X}^{2}(1+\exp (\lambda \widetilde{X}) I(\widetilde{X}>0)) \\
& \leq 1+c \lambda^{2}\left(\mathbb{E} X^{2}+\mathbb{E} X^{2} \exp (\lambda|\widetilde{X}|)\right)
\end{aligned}
$$

Let $\beta^{-\alpha}<\delta<\delta_{1}<1$ and $\lambda=\delta(\beta n)^{\alpha-1}$; then by $c_{r}$ inequality and $\mathbb{E}\left|X^{\prime}\right| \leq \mathbb{E}|X|<\infty$,

$$
\begin{aligned}
\lambda|\widetilde{X}| & \leq \lambda\left(\left|X^{\prime}\right|+\mathbb{E}\left|X^{\prime}\right|\right)^{\alpha}\left(\left|X^{\prime}\right|+\mathbb{E}\left|X^{\prime}\right|\right)^{1-\alpha} \\
& \leq \lambda\left(\left|X^{\prime}\right|^{\alpha}+c\right)\left(\left|X^{\prime}\right|^{1-\alpha}+c\right) \\
& \leq \delta(\beta n)^{\alpha-1}\left(|X|^{\alpha}+c\right)\left((\beta n)^{1-\alpha}+c\right) \\
& =\delta\left(|X|^{\alpha}+c\right)(1+o(1)) \leq \delta_{1}|X|^{\alpha}+c .
\end{aligned}
$$

By (5), $\mathbb{E} X^{2} \leq \mathbb{E} \exp \left(|X|^{\alpha}\right)<\infty$, and $\mathbb{E} X^{2} \exp \left(\delta_{1}|X|^{\alpha}\right) \leq$ $\mathbb{E} \exp \left(|X|^{\alpha}\right)<\infty$, therefore, by combination with (29) and (30), we get

$$
\begin{aligned}
\mathbb{E} \exp (\lambda \widetilde{X}) & \leq 1+c \lambda^{2}\left(\mathbb{E} X^{2}+c \mathbb{E} X^{2} \exp \left(\delta_{1}|X|^{\alpha}\right)\right) \\
& \leq 1+c \lambda^{2}=1+c n^{2(\alpha-1)} \\
& \leq \exp \left(c n^{2(\alpha-1)}\right) .
\end{aligned}
$$

Obviously, $X_{k}^{\prime}$ is increasing on $X_{k}$; thus, by Lemma 7(i), $\left\{X_{k}^{\prime} ; k \geq 1\right\}$ is also a sequence of ND random variables. Taking $a_{n}=\beta n$ and $\lambda=\delta(\beta n)^{\alpha-1}$ in Lemma 8 , for $1<\beta_{1}<$ $\delta \beta^{\alpha}$, we obtain

$$
\begin{aligned}
P\left(\max _{1 \leq k \leq n} S_{k}^{\prime}>n \beta\right) & \leq \exp (-\lambda \beta n) \sum_{k=1}^{n}(\mathbb{E} \exp (\lambda \widetilde{X}))^{k} \\
& \leq n \exp \left(-\lambda \beta n+c n^{2 \alpha-1}\right) \\
& =n \exp \left(\left(-\delta \beta^{\alpha}+c n^{-(1-\alpha)}\right) n^{\alpha}\right) \\
& \leq n \exp \left(-\beta_{1} n^{\alpha}\right) .
\end{aligned}
$$

Replacing $X_{k}^{\prime}$ by $-X_{k}^{\prime}$ and by the same argument as above,

$$
P\left(\max _{1 \leq k \leq n}\left(-S_{k}^{\prime}\right)>n \beta\right) \leq n \exp \left(-\beta_{1} n^{\alpha}\right)
$$

also holds. Hence,

$$
P\left(\max _{1 \leq k \leq n}\left|S_{k}^{\prime}\right|>n \beta\right) \leq 2 n \exp \left(-\beta_{1} n^{\alpha}\right)
$$

Thus, by combination with (27) and (28) and the fact that $\sum_{n=1}^{\infty}\left(n^{p} / \exp \left(c_{1} n^{\alpha}\right)\right)<\infty$ for all $c_{1}>0 p \in \mathbb{R}$, we obtain that, for $\beta_{2} \widehat{=} \min \left(\beta^{\alpha}, \beta_{1}\right)>1$,

$$
\begin{gathered}
\sum_{n=1}^{\infty} \exp \left(n^{\alpha}\right) n^{p} P\left(\max _{1 \leq k \leq n}\left|S_{k}\right|>n \beta\right) \\
\ll \sum_{n=1}^{\infty} \frac{n^{p+1}}{\exp \left(\left(\beta_{2}-1\right) n^{\alpha}\right)}<\infty .
\end{gathered}
$$

Hence, (24) holds. Next, we prove (25).

Let $x \geq n$. Replace $n$ by $x$ in $X^{\prime}$ and $X_{k}^{\prime}$. Using similar methods to those used in the proof of (27)-(34), there is $\beta_{2}>$ 1 such that

$$
P\left(\max _{1 \leq k \leq n}\left|S_{k}\right|>x \beta\right) \ll n \exp \left(-\beta_{2} x^{\alpha}\right)
$$


Thus,

$$
\begin{aligned}
& \int_{n}^{\infty} x^{q-1} P\left(\max _{1 \leq k \leq n}\left|S_{k}\right|>x \beta\right) \mathrm{d} x \ll n \int_{n}^{\infty} x^{q-1} \\
& \cdot \exp \left(-\beta_{2} x^{\alpha}\right) \mathrm{d} x \\
& \quad n \int_{n}^{\infty}\left(x^{q-1}-(q-\alpha)\left(\beta_{2} \alpha\right)^{-1} x^{q-1-\alpha}\right) \\
& \cdot \exp \left(-\beta_{2} x^{\alpha}\right) \mathrm{d} x \\
& \quad=n \int_{n}^{\infty}\left(\frac{x^{q-\alpha} \exp \left(-\beta_{2} x^{\alpha}\right)}{-\beta_{2} \alpha}\right)^{\prime} \mathrm{d} x \ll n^{q-\alpha+1} \\
& \cdot \exp \left(-\beta_{2} n^{\alpha}\right),
\end{aligned}
$$

and it implies that

$$
\begin{gathered}
\sum_{n=1}^{\infty} \exp \left(n^{\alpha}\right) n^{p} \int_{n}^{\infty} x^{q-1} P\left(\max _{1 \leq k \leq n}\left|S_{k}\right|>x \beta\right) \mathrm{d} x \\
\ll \sum_{n=1}^{\infty} \frac{n^{p+q+1-\alpha}}{\exp \left(\left(\beta_{2}-1\right) n^{\alpha}\right)}<\infty .
\end{gathered}
$$

That is, (25) holds.

Conversely, if (6) holds for $p=\alpha-q-2$ and some $\beta>0$, then, by $\mathbb{E}\left\{\max _{1 \leq k \leq n}\left|S_{k}\right|-\beta n\right\}_{+}^{q} \geq \beta^{q} \int_{0}^{n} q x^{q-1} P\left(\max _{1 \leq k \leq n}\left|S_{k}\right|\right.$ $-\beta n>\beta x) \mathrm{d} x \geq \beta^{q} n^{q} P\left(\max _{1 \leq k \leq n}\left|S_{k}\right|>2 \beta n\right)$, we have

$$
\sum_{n=1}^{\infty} \exp \left(n^{\alpha}\right) n^{\alpha-2} P\left(\max _{1 \leq k \leq n}\left|S_{k}\right|>2 \beta n\right)<\infty
$$

by combination with $\max _{1 \leq k \leq n}\left|X_{k}\right| \leq 2 \max _{1 \leq k \leq n}\left|S_{k}\right|$, it follows that

$$
\sum_{n=1}^{\infty} \frac{\exp \left(n^{\alpha}\right)}{n^{2-\alpha}} P\left(\max _{1 \leq k \leq n}\left|X_{k}\right|>4 \beta n\right)<\infty,
$$

and it implies that $P\left(\max _{1 \leq k \leq n}\left|X_{k}\right|>4 \beta n\right) \rightarrow 0, n \rightarrow \infty$. Thus, by Lemma 10, there is $c>0$ such that

$$
n P(|X|>4 \beta n) \leq c P\left(\max _{1 \leq k \leq n}\left|X_{k}\right|>4 \beta n\right) .
$$

Consequently, by (40),

$$
\sum_{n=1}^{\infty} \frac{\exp \left(n^{\alpha}\right)}{n^{1-\alpha}} P\left(\frac{|X|}{4 \beta}>n\right)<\infty,
$$

and, hence, $\mathbb{E} \exp \left(|X /(4 \beta)|^{\alpha}\right)<\infty$ from Lemma 9. This completes the proof of Theorem 3 .

Proof of Theorem 4. Let $X^{\prime}$ and $\widetilde{X}$ be defined as Theorem 3 . Let $\beta^{-1}<\delta<1$, and $\lambda=\delta \ln ^{-r}(\beta n)$; then,

$$
\begin{aligned}
\lambda|\widetilde{X}| & \leq \delta \ln ^{-r}(\beta n)\left(\left|X^{\prime}\right|+c\right) \leq \delta \ln ^{-r}\left(\left|X^{\prime}\right|\right)\left|X^{\prime}\right|+c \\
& \leq \delta|X| \ln ^{-r}(|X|)+c
\end{aligned}
$$

from $\left|X^{\prime}\right| \leq \beta n$ and $x \ln ^{-r} x$ being monotonically increasing on $x$.

Thus, by (8), $\mathbb{E} X^{2} \exp \left(\delta|X| \ln ^{-r}|X|\right) \ll \mathbb{E} \exp \left(|X| \ln ^{-r}|X|\right)$ $<\infty$; therefore, similar to (29), we have

$$
\mathbb{E} \exp (\lambda \widetilde{X}) \leq 1+c \ln ^{-2 r} n \leq \exp \left(c \ln ^{-2 r} n\right) .
$$

By Lemma 8 , for $1<\beta_{1}<\delta \beta<\beta$, we get

$$
\begin{aligned}
& P\left(\max _{1 \leq k \leq n} S_{k}^{\prime}>n \beta\right) \leq n \exp \left(-\lambda \beta n+c n \ln ^{-2 r} n\right) \\
& \quad=n \exp \left(-\delta \beta n \ln ^{-r}(\beta n)+c n \ln ^{-2 r} n\right) \\
& \quad \leq n \exp \left(-\beta_{1} n \ln ^{-r} n\right) .
\end{aligned}
$$

Replacing $X_{k}^{\prime}$ by $-X_{k}^{\prime}$ and by the same argument as above,

$$
P\left(\max _{1 \leq k \leq n}\left(-S_{k}^{\prime}\right)>n \beta\right) \leq n \exp \left(-\beta_{1} n \ln ^{-r} n\right)
$$

also holds. Hence,

$$
P\left(\max _{1 \leq k \leq n}\left|S_{k}^{\prime}\right|>n \beta\right) \leq 2 n \exp \left(-\beta_{1} n \ln ^{-r} n\right) .
$$

On the other hand, from (8) and the Markov inequality,

$$
\begin{aligned}
P(|X|>n \beta) & \ll \exp \left(-\beta n \ln ^{-r}(\beta n)\right) \\
& \leq \exp \left(-\beta_{1} n \ln ^{-r} n\right) .
\end{aligned}
$$

Hence, together with (27),

$$
\begin{gathered}
\sum_{n=1}^{\infty} \exp \left(n \ln ^{-r} n\right) n^{p} P\left(\max _{1 \leq k \leq n}\left|S_{k}\right|>n \beta\right) \\
\ll \sum_{n=1}^{\infty} \frac{n^{p+1}}{\exp \left(\left(\beta_{1}-1\right) n \ln ^{-r} n\right)}<\infty .
\end{gathered}
$$

Similar to (36) and the above discussion, for $x \geq n$,

$$
P\left(\max _{1 \leq k \leq n}\left|S_{k}\right|>x \beta\right) \ll n \exp \left(-\beta_{1} x \ln ^{-r} x\right) .
$$

Thus,

$$
\begin{aligned}
& \int_{n}^{\infty} x^{q-1} P\left(\max _{1 \leq k \leq n}\left|S_{k}\right|>x \beta\right) \mathrm{d} x \ll n \int_{n}^{\infty} x^{q-1} \\
& \cdot \exp \left(-\beta_{1} x \ln ^{-r} x\right) \mathrm{d} x \sim n \int_{n}^{\infty}\left(x^{q-1}-r x^{q-1} \ln ^{-1} x\right. \\
& \left.-(q-1) \beta_{1}^{-1} x^{q-2} \ln ^{r} x-r \beta_{1}^{-1} x^{q-2} \ln ^{r-1} x\right) \\
& \cdot \exp \left(-\beta_{1} x \ln ^{-r} x\right) \mathrm{d} x \\
& =n \int_{n}^{\infty}\left(\frac{x^{q-1} \ln ^{r} x \exp \left(-\beta_{1} x \ln ^{-r} x\right)}{-\beta_{1}}\right)^{\prime} \mathrm{d} x \\
& \ll n^{q} \ln ^{r} n \exp \left(-\beta_{1} n \ln ^{-r} n\right),
\end{aligned}
$$


and it implies that

$$
\begin{aligned}
& \sum_{n=1}^{\infty} \exp \left(n \ln ^{-r} n\right) n^{p} \int_{n}^{\infty} x^{q-1} P\left(\max _{1 \leq k \leq n}\left|S_{k}\right|>x \beta\right) \mathrm{d} x \\
& \ll \sum_{n=1}^{\infty} \frac{n^{p+q} \ln ^{r} n}{\exp \left(\left(\beta_{1}-1\right) n \ln ^{-r} n\right)}<\infty .
\end{aligned}
$$

Thus, by combination with (23) and (49), (9) holds.

Conversely, by (18), using similar methods to those used in the proof of (7), we can get (10). This completes the proof of Theorem 4.

\section{Competing Interests}

The authors declare that they have no competing interests.

\section{Authors' Contributions}

Qunying Wu conceived the study and drafted, completed, read, and approved the final paper. Yuanying Jiang conceived the study and completed, read, and approved the final paper.

\section{Acknowledgments}

This work was supported by the National Natural Science Foundation of China (11361019) and the Support Program of the Guangxi China Science Foundation (2015GXNSFAA139008).

\section{References}

[1] N. Ebrahimi and M. Ghosh, "Multivariate negative dependence," Communications in Statistics - Theory and Methods, vol. 10, no. 4, pp. 307-337, 1981.

[2] E. L. Lehmann, "Some concepts of dependence," Annals of Mathematical Statistics, vol. 37, no. 5, pp. 1137-1153, 1966.

[3] K. Joag-Dev and F. Proschan, "Negative association of random variables with applications," The Annals of Statistics, vol. 11, no. 1, pp. 286-295, 1983.

[4] A. Bozorgnia, R. F. Patterson, and R. L. Taylor, "Limit theorems for ND r.v.s,” Tech. Rep., University of Georgia, Athens, Greece, 1993.

[5] Q. Y. Wu and Y. Y. Jiang, "The strong consistency of $M$ estimator in a linear model for negatively dependent random samples," Communications in Statistics. Theory and Methods, vol. 40, no. 3, pp. 467-491, 2011.

[6] V. Fakoor and H. A. Azarnoosh, "Probability inequalities for sums of negatively dependent random variables," Pakistan Journal of Statistics, vol. 21, no. 3, pp. 257-264, 2005.

[7] N. Asadian, V. Fakoor, and A. Bozorgnia, "Rosen-thal's type inequalities for negatively orthant dependent random variables," Journal of the Iranian Chemical Society, vol. 5, no. 1-2, pp. 66-75, 2006.

[8] Q. Y. Wu, "Complete convergence for negatively dependent sequences of random variables," Journal of Inequalities and Applications, vol. 2010, Article ID 507293, 10 pages, 2010.
[9] X. J. Wang, X. Deng, L. L. Zheng, and S. H. Hu, "Complete convergence for arrays of rowwise negatively superadditivedependent random variables and its applications," Statistics, vol. 48, no. 4, pp. 834-850, 2014.

[10] C.-C. Liu, M.-L. Guo, and D.-J. Zhu, "Equivalent conditions of complete convergence for weighted sums of sequences of extended negatively dependent random variables," Communications in Mathematical Research, vol. 31, no. 1, pp. 40-50, 2015.

[11] Y. S. Chow, "On the rate of moment convergence of sample sums and extremes," Bulletin of the Institute of Mathematics. Academia Sinica, vol. 16, no. 3, pp. 177-201, 1988.

[12] P. Y. Chen and D. C. Wang, "Complete moment convergence for sequence of identically distributed-mixing random variables," Acta Mathematica Sinica, vol. 26, no. 4, pp. 679-690, 2010.

[13] A. Gut and U. Stadtmüller, "An intermediate Baum-Katz theorem," Statistics \& Probability Letters, vol. 81, no. 10, pp. 14861492, 2011.

[14] S. H. Sung, "Complete qth moment convergence for arrays of random variables," Journal of Inequalities and Applications, vol. 2013, article 24, 2013.

[15] M. L. Guo, "Equivalent conditions of complete moment convergence of weighted sums for $\phi$-mixing sequence of random variables," Communications in Statistics. Theory and Methods, vol. 43, no. 10-12, pp. 2527-2539, 2014.

[16] D. H. Qiu and P. Y. Chen, "Complete and complete moment convergence for weighted sums of widely orthant dependent random variables," Acta Mathematica Sinica, vol. 30, no. 9, pp. 1539-1548, 2014.

[17] D. H. Qiu and P. Y. Chen, "Complete moment convergence for i.i.d. random variables," Statistics \& Probability Letters, vol. 91, pp. 76-82, 2014.

[18] Y. B. Wang, Y. W. Li, and Q. W. Gao, "On the exponential inequality for acceptable random variables," Journal of Inequalities and Applications, vol. 2011, article 40, 10 pages, 2011.

[19] Q. Y. Wu, "Sufficient and necessary conditions of complete convergence for weighted sums of PNQD random variables," Journal of Applied Mathematics, vol. 2012, Article ID 104390, 10 pages, 2012. 


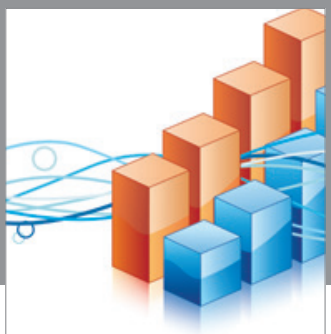

Advances in

Operations Research

vatem alat4

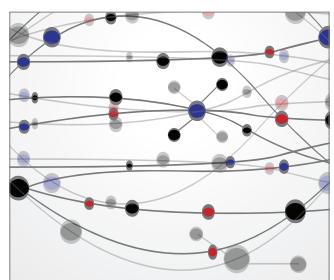

\section{The Scientific} World Journal
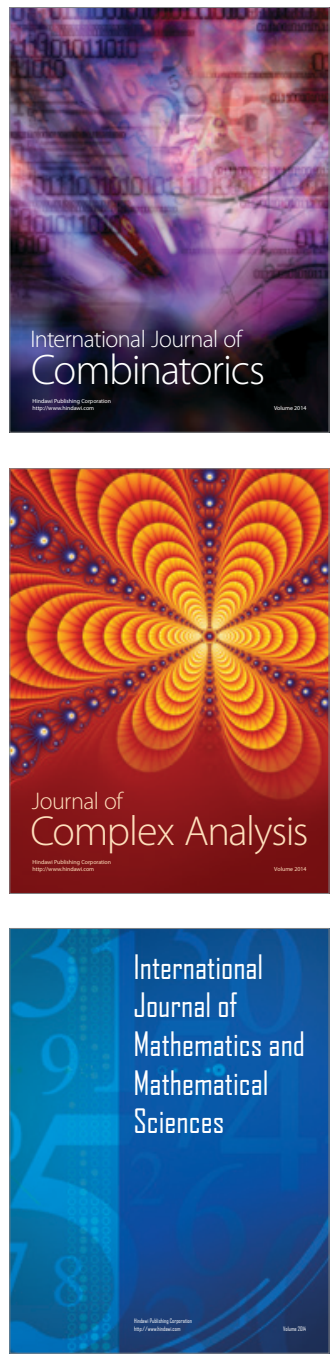
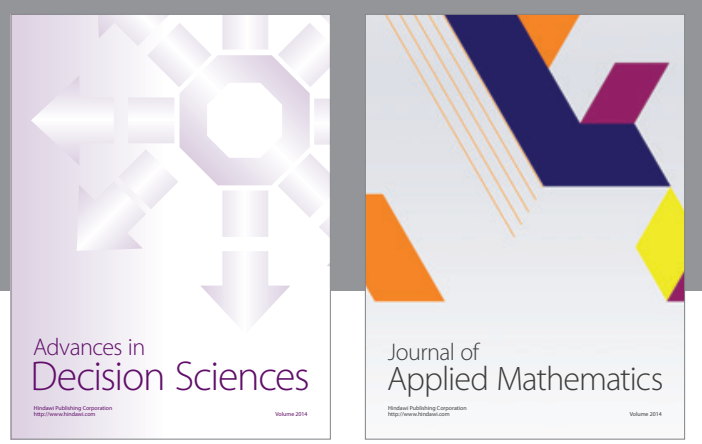

Algebra

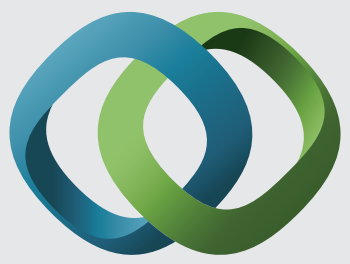

\section{Hindawi}

Submit your manuscripts at

http://www.hindawi.com
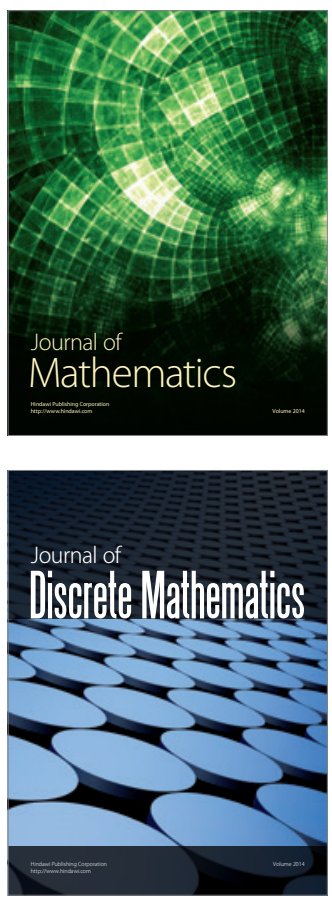

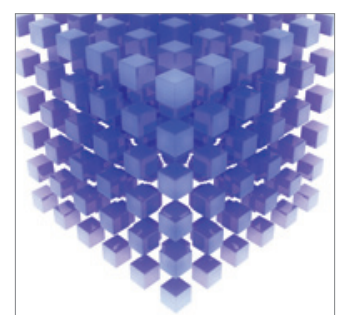

Mathematical Problems in Engineering
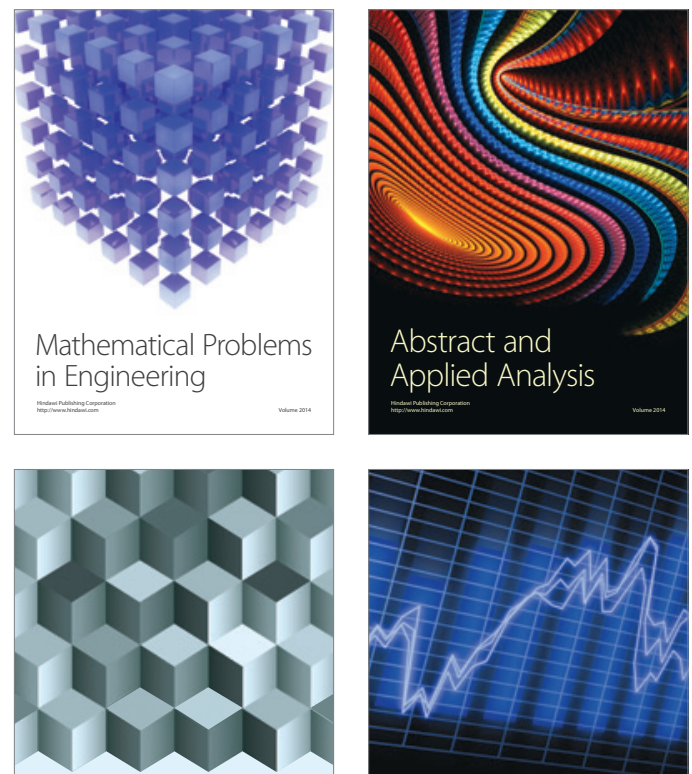

Journal of

Function Spaces

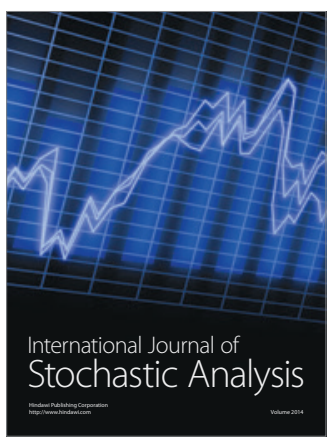

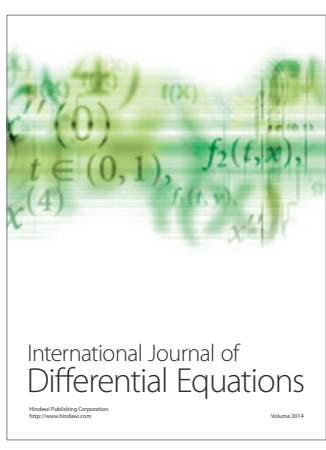
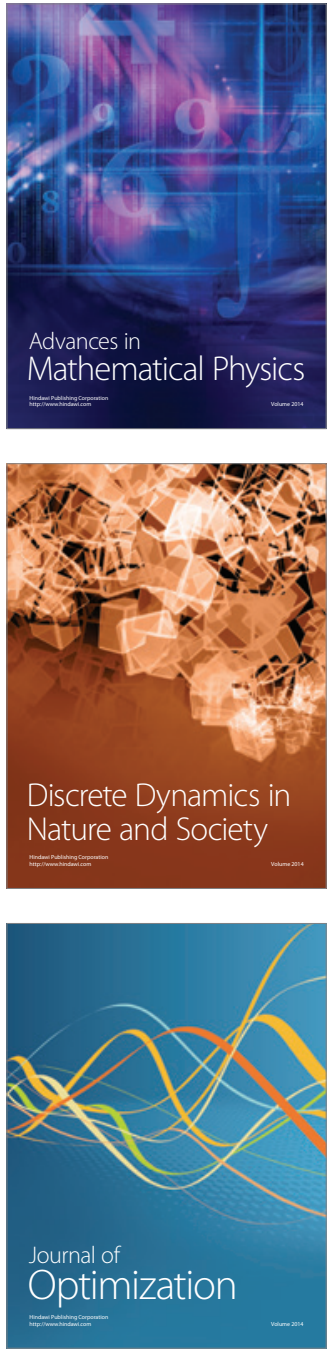